\title{
Proteomic analysis of the temporal expression of bovine milk proteins during coliform mastitis and label-free relative quantification
}

\author{
J. L. Boehmer, ${ }^{*}{ }^{1}$ J. L. Ward, ${ }^{*}$ R. R. Peters, $\dagger$ K. J. Shefcheck, $\ddagger$ M. A. McFarland, $\ddagger$ and D. D. Bannerman $\S^{2}$ \\ *US Food and Drug Administration Center for Veterinary Medicine, Laurel, MD 20708 \\ †Department of Animal and Avian Sciences, University of Maryland College Park, 20742 \\ fUS Food and Drug Administration Center for Food Safety and Applied Nutrition, College Park, MD 20742 \\ §Bovine Functional Genomics Laboratory, USDA—Agricultural Research Service, Beltsville, MD 20705
}

\begin{abstract}
The discovery of biomarkers in milk indicative of local inflammation or disease in the bovine mammary gland has been hindered by the extreme biological complexity of milk, the dynamic range of proteins in the matrix that renders the identification of low-abundance proteins difficult, and the challenges associated with quantifying changes during disease in the abundance of proteins for which no antibody exists. The objectives of the current study were to characterize the temporal expression of milk proteins following Escherichia coli challenge and to evaluate change in relative abundance of identified proteins using a liquid chromatography coupled with tandem mass spectrometry (LC-MS/ MS) label-free semiquantitative approach. Liquid chromatography-MS/MS conducted on whey from milk samples collected just before infusion with $E$. coli and at $12,18,24,36,48$, and $60 \mathrm{~h}$ following infection resulted in the identification of the high- to mediumabundance proteins $\alpha_{\mathrm{S1}^{-}}, \alpha_{\mathrm{S}_{2}}-\beta$-, and $\kappa$-caseins and the whey proteins serum albumin, $\beta$-lactoglobulin, and $\alpha$-lactalbumin. Additionally, a select number of lower abundance markers of inflammation were also identified, including lactoferrin, transferrin, apolipoprotein AI, fibrinogen, glycosylation-dependent cell adhesion molecule-1, peptidoglycan recognition receptor protein, and cyclic dodecapeptide-1. Normalized peptide counts for each protein identified were used to evaluate temporal changes in milk proteins following infection. For comparison with relative protein abundance determined using proteomic-based methods, changes in serum albumin, lactoferrin, and transferrin in milk during disease were also measured using ELISA. Label-free, proteomicbased quantification revealed relative changes in milk proteins that corresponded to expression profiles gener-
\end{abstract}

Received June 29, 2009

Accepted October 13, 2009.

${ }^{1}$ Corresponding author: jamie.boehmer@fda.hhs.gov

${ }^{2}$ Current affiliation: United States Department of Veterans Affairs, Office of Research Oversight, Washington, DC 20420. ated by ELISA. The results indicate that label-free LCMS/MS methods are a viable means of tracking changes in relative protein abundance in milk during disease. Despite the identification of primarily abundant milk proteins, the results indicate that, with further refinement, LC-MS/MS could be used to evaluate temporal changes in proteins related to host response for which no antibody or ELISA currently exists.

Key words: bovine milk proteome, liquid chromatography-tandem mass spectrometry, coliform mastitis, label-free quantification

\section{INTRODUCTION}

The use of proteomics to characterize the bovine milk proteome is a relatively new approach in dairy cattle research that has to date involved only a limited number of endeavors. Efforts to characterize proteins present in the whey and milk fat globular membrane fractions from the milk of healthy cows have not yet succeeded in completely characterizing the bovine milk proteome, but have contributed to our knowledge of both highand low-abundance proteins present in bovine milk (Reinhardt and Lippolis, 2006, 2008; Fong et al., 2008). Additionally, recent proteomic analyses of milk from healthy cows and cows with clinical mastitis have been successful in identifying differentially expressed proteins and protein biomarkers of host response present in bovine milk during disease (Hogarth et al., 2004; Smolenski et al., 2007; Boehmer et al., 2008).

More complete annotation of the bovine milk proteome and the assessment of changes in milk protein expression during disease remains hindered by the biological complexity and extreme dynamic range of proteins present in milk, as well as the number of genetic variants and numerous posttranslational modifications of milk proteins (O'Donnell et al., 2004; Lippolis and Reinhardt, 2008). The identification of extremely low abundance proteins in bovine milk during disease would augment current knowledge of the bovine milk proteome and enable both the characterization of pathways that occur during lactation and the potential identification 
of biomarkers related to host response, but several fundamental challenges remain associated with biomarker discovery in milk (O'Donnell et al., 2004; Gagnaire et al., 2009). Two main challenges are 1) refining fractionation methodologies and reducing sample complexity to mine the deep proteome of bovine milk where diagnostically significant biomarkers are most likely to reside (Garbis et al., 2005; Simpson et al., 2009), and 2) developing methods to accurately quantify changes in protein abundance so that identified biomarkers could be used to evaluate disease progression or the efficacy of a drug treatment (Old et al., 2005; Mueller et al., 2008; Simpson et al., 2009). Because of the specific importance of the latter issue for a biomarker or pattern of biomarkers to be considered for validation studies, the quantification of changes in the abundance of proteins in a given biological sample has garnered a significant amount of attention in the field of proteomics in recent years (Mueller et al., 2008; Pavelka et al., 2008; Simpson et al., 2009).

In addition to the complexity of milk, proteomic analyses of biomarkers of disease in bovine milk (Hogarth et al., 2004; Smolenski et al., 2007; Boehmer et al., 2008) have suffered from the use of too few biological replicates as well as the analysis of only a limited number of milk samples collected over time during the course of clinical mastitis. Likewise, current knowledge of biomarkers of coliform mastitis pertains almost exclusively to the expression profiles of cytokines in milk following Escherichia coli infection or LPS challenge derived using ELISA (reviewed in Bannerman, 2009). Contrary to proteomic methodologies, ELISA is designed to measure only 1 protein at a time and is limited by antibody development and availability. Proteomics, on the other hand, is theoretically unlimited in the number of proteins that can be studied in a given sample and is not constrained by the need for speciesspecific antibodies for quantification.

Accurate quantification of the abundance of individual proteins identified in complex biological samples using proteomic strategies is, however, an emerging research area. A popular means of quantifying proteins in a complex biological sample has been 2-dimensional gel electrophoresis, but the intact protein approach using electrophoresis and quantification via densitometry is limited in sensitivity and problematic for proteins that are insoluble or have either a very high or low molecular weight (Old et al., 2005). An alternative approach is bottom up or shotgun proteomics, which involves the digestion of proteins in mixtures and subsequent analysis of resultant peptides using liquid chromatography coupled with tandem mass spectrometry (LC-MS/MS).

Several labeling strategies have been used in conjunction with LC-MS/MS analyses for the purpose of quan- tifying changes in protein abundance, but the labeling of intact proteins or peptides can be cost limiting, is not amenable to all biological matrices, and frequently requires pairwise comparisons analysis between samples that renders retrospective assessments impossible (Old et al., 2005; Mueller et al., 2008; Simpson et al., 2009). Accordingly, recent trends have been toward label-free relative quantification based on the linear relationship between sampling statistics observed using LC-MS/ MS and relative protein abundance. Sampling statistics evaluated as potential measures of relative protein abundance include 1) the mean peak area intensity of all peptides identified for an individual protein in a complex sample (Bondarenko et al., 2002; Old et al., 2005); 2) the peptide count, or total number of peptides identified from a given protein in an LC-MS/MS experiment (Gao et al., 2003; Zhang et al., 2006); and 3) spectral counts, or the total number of tandem mass spectra generated on a given peptide in an LC-MS/MS experiment (Liu et al., 2004; Old et al., 2005; Zybailov et al., 2005). Peak area refers to the intensity of peptide ions in a mass spectrum, whereas the primary distinction between a spectral count and a peptide count is that the latter refers to the number of peptides identified for a given protein in an LC-MS/MS experiment whereas a spectral count details the actual number of times an individual peptide is selected for MS/MS collision-induced dissociation (CID) and is therefore the frequency by which a peptide is identified (Old et al., 2005; Zhang et al., 2006; Mueller et al., 2008).

Quantification of milk protein modulation during mastitis has never been attempted using proteomic strategies. A first step toward establishing reliable biomarkers of coliform mastitis for use in monitoring disease progression or evaluating drug efficacy would be to investigate the viability of using LC-MS/MS proteomic strategies to quantify changes in the relative abundance of milk proteins during disease. Additionally, the comparison of semiquantitative LC-MS/ MS methods for determining relative abundance with expression profiles generated using an ELISA would aid in validating LC-MS/MS as a strategy to quantify changes in proteins for which no antibody has been developed. Also, using samples collected over the course of infection from several biological replicates could aid in the discovery of a reproducible biomarker or pattern of biomarkers specific to mastitis, and the time frame following infection that the biomarker or biomarkers could be accurately monitored. Accordingly, the goals of the current study were to profile temporal expression of proteins in bovine milk samples collected over a time course following infection with $E$. coli using LC-MS/MS and to use peptide counts, a label-free semiquantitative approach, to quantify relative changes in identified 
proteins. A further objective was to compare observed trends in protein modulation detected using LC-MS/ MS with quantification using ELISA to investigate the accuracy of using a nonantibody-based semiquantitative approach to screen for biologically relevant proteins expressed in milk during coliform mastitis.

\section{MATERIALS AND METHODS}

\section{Cows}

Eight clinically healthy multiparous Holstein cows in middle to late lactation $(211 \pm 12$ DIM $)$ were selected on the basis of milk SCC of $<200,000$ cells $/ \mathrm{mL}$ and no history of treatment for mastitis over the past 6 mo. The care and use of all animals in this study were approved by the University of Maryland's Animal Care and Use Committee.

\section{Determination of Milk SCC}

To quantify somatic cells, milk samples were heated to $60^{\circ} \mathrm{C}$ for $15 \mathrm{~min}$ and subsequently maintained at $40^{\circ} \mathrm{C}$ until counted on an automated cell counter (Bentley Somacount 150; Bentley Instruments Inc., Chaska, MN).

\section{E. coli Challenge and Whey Preparation}

Preparation of the inoculum and experimental infection were performed as described previously (Bannerman et al., 2004). Following overnight incubation, 1 $\mathrm{mL}$ of inoculum was serially diluted in PBS and plated on trypticase soy agar plates. Plates were incubated overnight at $37^{\circ} \mathrm{C}$ and the stock solution was maintained at $4^{\circ} \mathrm{C}$. After determination of the stock culture concentration based on colony counts, the stock culture was diluted in PBS to a final concentration of approximately $40 \mathrm{cfu} / \mathrm{mL}$.

Baseline milk samples were collected aseptically from the right front quarter of each of the 8 cows the evening before and the morning of challenge. Following morning milking, the right front quarter of each of the 8 cows was infused with $2 \mathrm{~mL}$ of $40 \mathrm{cfu} / \mathrm{mL}$ of $E$. coli (approximately $80 \mathrm{cfu}$ ). Milk samples were collected aseptically at $12,18,24,36,48$, and $60 \mathrm{~h}$ following challenge. For the preparation of whey, milk samples were centrifuged at $3,000 \times g$ at $4^{\circ} \mathrm{C}$ for $30 \mathrm{~min}$ and the fat layer was removed. The skim milk was transferred to a clean tube, centrifuged again $20,000 \times g$ at $4^{\circ} \mathrm{C}$ for $60 \mathrm{~min}$, and the translucent supernatant was collected, aliquoted into sterile $1.5-\mathrm{mL}$ microcentrifuge tubes, and stored at $-80^{\circ} \mathrm{C}$.

\section{ELISA for BSA, Lactoferrin, and Transferrin}

Milk levels of BSA were determined using a commercially available ELISA kit (Bethyl Laboratories
Inc., Montgomery, TX) with slight modifications as described previously (Bannerman et al., 2003). Concentrations of lactoferrin and transferrin in milk were determined using commercially available ELISA kits (Bethyl Laboratories Inc.) as described previously for BSA (Bannerman et al., 2003) with the slight modification that the wash and blocking buffers used were 50 $\mathrm{m} M$ tris-buffered saline with $0.05 \%$ Tween and $50 \mathrm{mM}$ tris-buffered saline with $1 \%$ BSA, respectively, $\mathrm{pH} 8.0$, for both assays.

Absorbance for all ELISA was read at $450 \mathrm{~nm}$ on a microplate reader (SpectraMax Plus; Molecular Devices, Sunnyvale, CA), and the concentrations of BSA, transferrin, and lactoferrin were calculated by extrapolating from a standard curve using SoftMax Pro software (v. 5.2; Molecular Devices).

\section{In-Solution Digests}

The protein content of all of the whey fractions from each cow $(\mathrm{n}=56)$ was determined using a bicinchoninic acid assay (Pierce Biotechnology, Rockford, IL) using BSA as a standard. Approximately $500 \mu \mathrm{g}$ of each whey sample was diluted in $0.5 \mathrm{M}$ triethylammonium bicarbonate (Sigma Aldrich, St. Louis, MO)/6 $M$ urea buffer to a final volume of $100 \mu \mathrm{L}$. Samples were reduced in a final concentration of $5 \mathrm{mM}$ Tris(2-carboxyethyl)phosphine (Promega, Madison, WI) for $1 \mathrm{~h}$ at $37^{\circ} \mathrm{C}$. Samples were alkylated in a final concentration of $10 \mathrm{~m} M$ iodoacetamide for $1 \mathrm{~h}$ at room temperature in the dark. Prior to the addition of trypsin, samples were diluted with distilled water to reduce urea to a final concentration of $<1 M$. Approximately $5 \mu \mathrm{g}$ of sequencing-grade modified trypsin (Promega) was added to each sample to achieve a 1:100 enzyme:protein ratio, and digestion was carried out for $16 \mathrm{~h}$ in a $37^{\circ} \mathrm{C}$ water bath. Samples were acidified following digestion by the addition of acetic acid to a final concentration of $0.5 \%$.

\section{LC-MS/MS Analyses}

Following trypsin digestion of approximately 500 $\mu \mathrm{g}$ of each whey sample, 1-dimensional LC-MS/MS analyses were carried out by injecting $10 \mu \mathrm{L}$ of the total reaction volume of $500 \mu \mathrm{L}$ of each whey digest into an Acquity ultra-pressure liquid chromatography instrument (Waters, Milford, MA) coupled to a quadrupole time-of-flight Q-Tof Premier mass spectrometer (Waters). Comparisons were made between samples based on the loading of an equal amount of sample onto the ultra-pressure liquid chromatography column. Peptides were loaded onto a Symmetry NanoEase $300 \mu \mathrm{m} \times$ $150 \mathrm{~mm} \mathrm{C}_{18}$ reverse-phase HPLC column (Waters). The mobile phase consisted of $0.1 \%$ formic acid in water 
(solvent A) and $0.1 \%$ formic acid in acetonitrile (solvent B). The gradient was $100 \% \mathrm{~A}$ for 5 min and then increased linearly to $60 \%$ solvent B over 70 min at a flow rate of $20 \mu \mathrm{L} / \mathrm{min}$. The quadrupole time-of-flight mass spectrometer was equipped with an electro-spray ionization source and was operated in positive ion mode with a capillary voltage of $3.0 \mathrm{kV}$. Spectra were acquired for $70 \mathrm{~min}$ in data-dependent MS/MS mode. The MS survey scans were conducted on peptides from a mass-to-charge ratio $(\mathrm{m} / \mathrm{z})$ of 400 to 2,000 and were a maximum of $0.6 \mathrm{~s}$ in duration. The 5 most intense ions in each MS survey scan were subjected to MS/MS by CID. Following MS/MS, precursor ions were excluded from MS/MS for $10 \mathrm{~s}$.

\section{Database Searching and Protein Identification}

Mass spectral peak lists were extracted using Mascot Distiller and resulting peak lists were searched with the Mascot search engine (v. 2.1.0; Matrix Sciences, London, UK) against the UniprotKB Swiss-Prot protein sequence library. The following search parameters were applied: other mammalia species, trypsin enzyme, one allowed missed cleavage, carbamidomethylation fixed modification, methionine oxidation variable modification, precursor ion mass tolerance of $\pm 0.3 \mathrm{Da}$, and a fragment ion mass tolerance of $\pm 0.3 \mathrm{Da}$. The number of sequences searched in the other mammalia taxonomy was 10,635. Only peptides with Mascot expect scores less than 0.05 and with a Mascot ion score equal or greater to the identity threshold (at $P<0.05$ ) were considered for protein identification.

\section{Label-Free Quantification}

Label-free quantification was determined using the number of peptides identified per protein, or peptide counts (Gao et al., 2003; Zhang et al., 2006). The peptide counts for each identified protein were extracted from Mascot data files using the software program MassSieve (Slotta et al., 2007). Peptide counts were normalized relative to the total number of peptides identified in samples from all 8 cows at each sampling time point as described previously (McFarland et al., 2008). The average of the normalized values for the peptide counts across all 8 cows was calculated for each identified protein, and averages were expressed graphically as mean \pm standard error. To measure differences in protein abundance across time points, the fold change between normalized peptide counts before and after challenge was calculated. Changes in relative abundance of proteins across the time points following infection with $E$. coli were considered relevant if the difference between pre- and postchallenge normalized abundance factors was a 3-fold or greater number (McFarland et al., 2008; Mosley et al., 2009). The 3 -fold or greater level of significance was applied only to proteins that met the arbitrary threshold of greater than 5 peptide assignments per time point to avoid potential overestimation of fold changes in relative abundance of proteins with limited peptide assignments. For proteins not present in milk before challenge, fold changes were measured relative to the normalized peptide counts calculated at the time point at which the protein was first detected.

\section{Statistical Analysis}

Repeated-measures ANOVA was performed on ELISA data using the PROC MIXED model with Dunnett post hoc pairwise comparisons (SAS v. 9.1; SAS Institute, Cary, NC) to compare pre- and postinfection concentrations of the proteins BSA, lactoferrin, and transferrin. Means and standard errors for all ELISA and label-free quantification data were calculated and graphed using SigmaPlot (v. 11.0; Systat Software Inc., Chicago, IL). Milk SCC were log-transformed to milk SCS. For all statistical analyses, a value of $P<0.05$ for the comparisons was considered to represent a significant difference between groups.

\section{RESULTS}

\section{Milk Proteins Identified in Whey Before and Following Infection with E. coli}

Proteomic analyses of bovine milk proteins following infection with $E$. coli, using peptide counts as an estimation of relative protein abundance, revealed the modulation of several milk proteins as well as the appearance of proteins not present in milk before infection. Both prechallenge whey samples and whey samples collected at time points following experimental induction of coliform mastitis were characterized by the presence of the abundant milk proteins $\alpha_{\mathrm{S}^{-}} \mathrm{CN}, \alpha_{\mathrm{S}^{-}} \mathrm{CN}, \beta-\mathrm{CN}$, $\kappa-\mathrm{CN}, \beta-\mathrm{LG}, \alpha-\mathrm{LA}$, and BSA (Table 1). Although at lower frequencies, lactoferrin and glycosylation-dependent cell adhesion molecule- 1 were also identified in the milk of healthy cows and cows with mastitis (Table 2). Proteins identified only in mastitic whey samples included transferrin and some extremely low abundance proteins detected in fewer than 8 cows across different time points, including apolipoprotein A-I, fibrinogen, peptidoglycan recognition receptor protein (PGRP), and cyclic dodecapeptide (cathelicidin-1).

Peptide counts for the abundant whey proteins $\beta-\mathrm{LG}$ and $\alpha-\mathrm{LA}$ and the $\mathrm{CN}$ remained relatively consistent across all time points sampled in all biological replicates, whereas peptide counts for BSA increased drasti- 
Table 1. Average peptide counts for abundant milk proteins

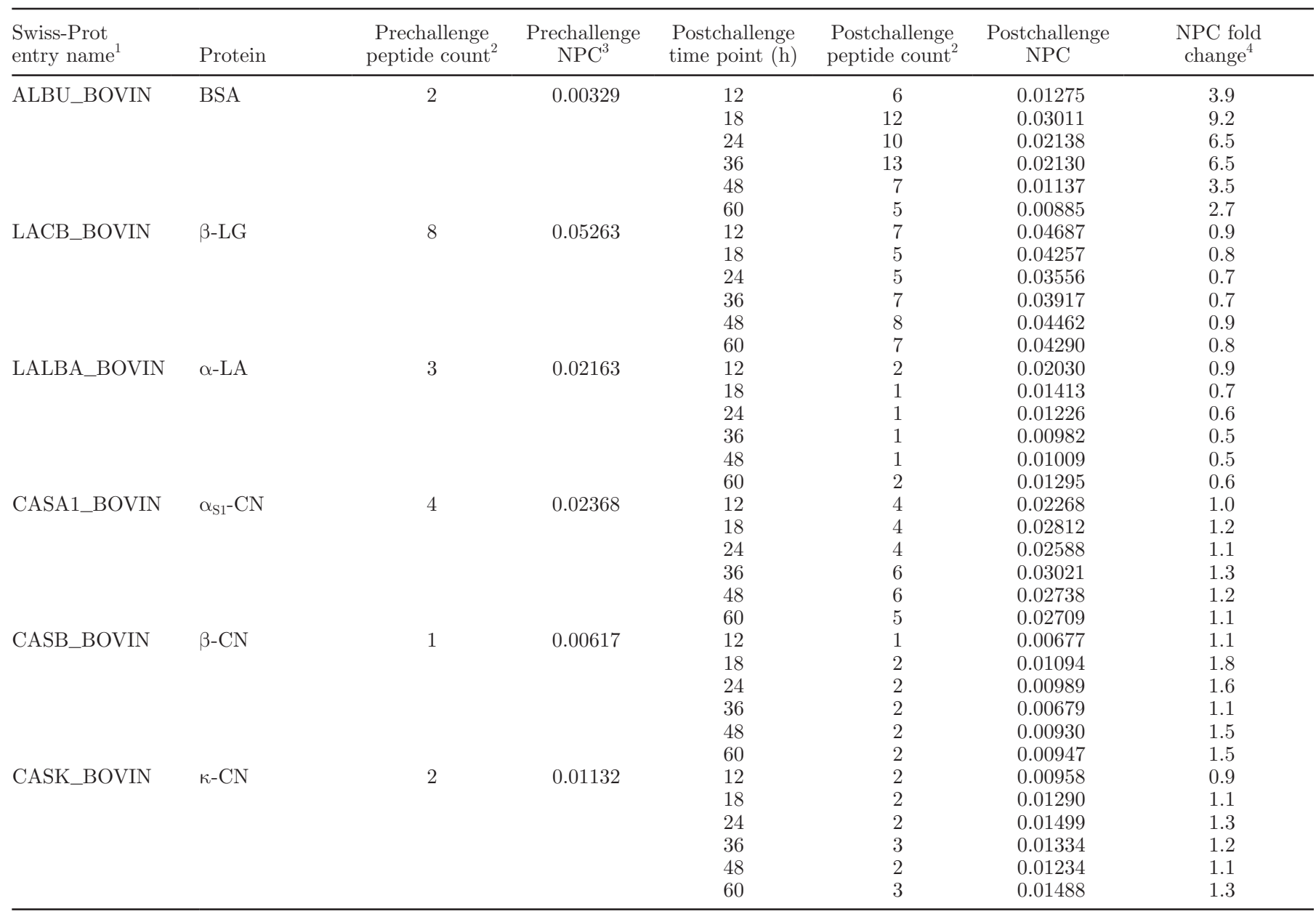

${ }^{1}$ Swiss-Prot $=$ UniprotKB Swiss-Prot protein sequence library (http://ca.expasy.org/sprot/).

${ }^{2}$ Peptide counts for each protein presented as an average for all 8 cows.

${ }^{3}$ Normalized peptide count.

${ }^{4}$ Fold change relative to prechallenge levels.

cally following infection relative to prechallenge levels (Supplemental Table 1; available online at http://www. journalofdairyscience.org/). Peptide counts for the protein lactoferrin increased toward the end of the time course analyzed, with peptide counts doubling from 48 to $60 \mathrm{~h}$ following infection. Conversely, peptide counts for transferrin were increased relative to prechallenge at $12 \mathrm{~h}$ following infection, but were below the level of detection in 48- or 60-h samples (Supplemental Table 1; available online at http://www.journalofdairyscience. org/). The only protein with sufficient peptide counts to meet the cut-off criteria for fold change assessment was BSA, which had normalized peptide counts that were 3 -fold greater than prechallenge levels at 12, 18, 24,36 , and $48 \mathrm{~h}$ following infection.

Spectral counts of peptides from abundant milk proteins were likewise assessed and found to be consistent with peptide counts in terms of temporal expression patterns and fold changes relative to prechallenge levels for each protein (Supplemental Table 2; available online at http://www.journalofdairyscience.org/). The limited number of peptide assignments per protein resulted in the underestimation of spectral counts for each peptide identified, however, and thus estimation of changes in the relative abundance of milk proteins during coliform mastitis was accomplished using peptide counts in lieu of spectral counts.

\section{ELISA Quantification of BSA, Lactoferrin, and Transferrin}

Increased permeability of the blood-milk barrier, a hallmark of coliform mastitis, was apparent by $18 \mathrm{~h}$ following infection as evidenced by increases in the vascular-derived proteins BSA and transferrin. The BSA measured in milk from quarters inoculated with $E$. coli exhibited significant 
Table 2. Average number of peptide counts for low-abundance milk proteins

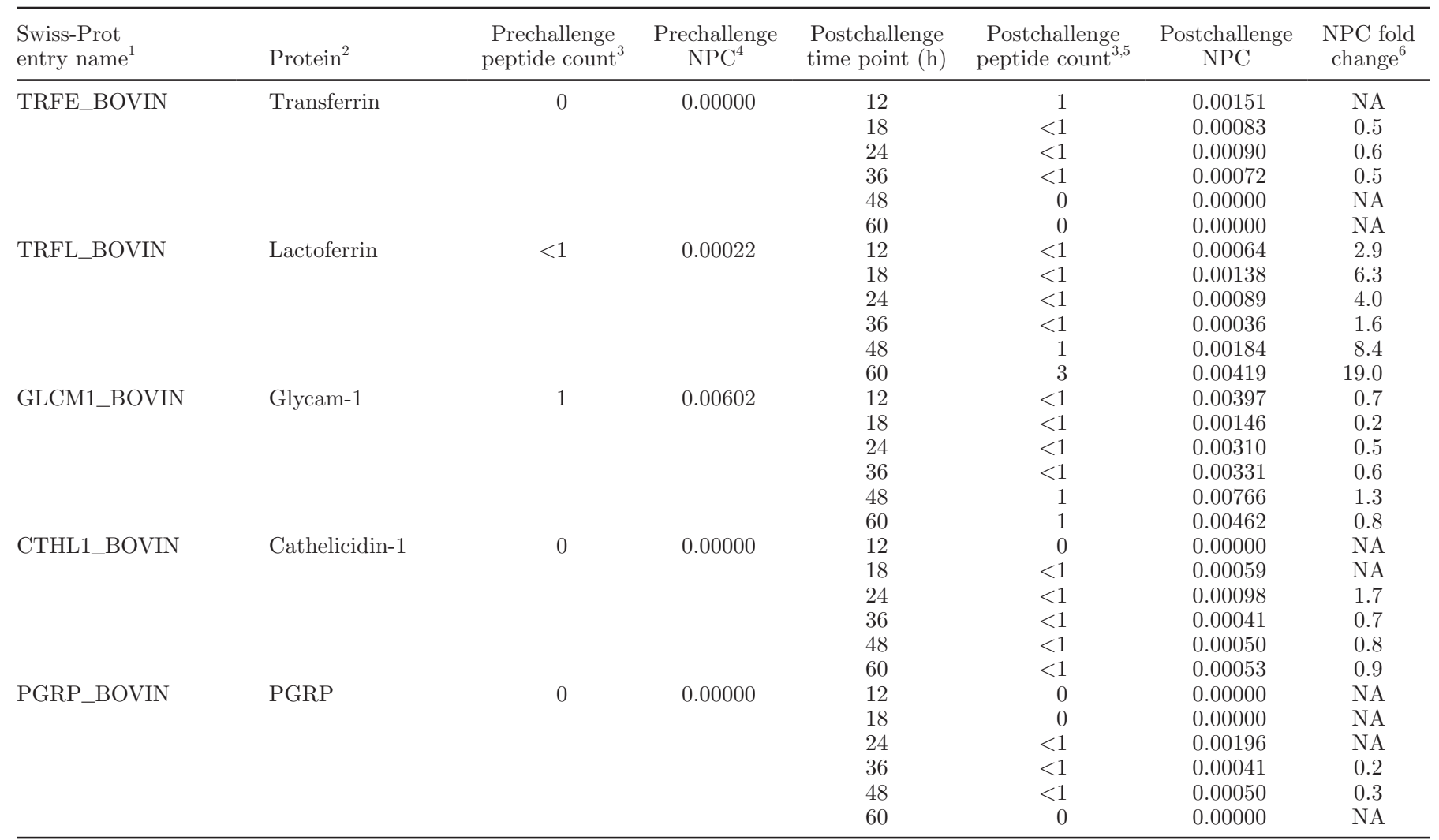

${ }^{1}$ Swiss-Prot = UniprotKB Swiss-Prot protein sequence library (http://ca.expasy.org/sprot/).

${ }^{2}$ Glycam- 1 = glycosylation-dependent cell adhesion molecule-1; cathelicidin-1 = cyclic dodecapeptide; PGRP = peptidoglycan recognition receptor protein.

${ }^{3}$ Peptide counts for each protein presented as an average for all 8 cows.

${ }^{4}$ Normalized peptide count.

${ }^{5}$ Number of peptides $<1$ indicates that the peptide was detected in samples from fewer than 8 cows.

${ }^{6}$ Fold change relative to prechallenge levels, or the first time point detected. NA = not applicable.

$(P<0.05)$ differences in concentration at 18 and $24 \mathrm{~h}$ following infection when compared with levels detected in milk before infection (Figure 1A). The BSA levels in milk from cows with coliform mastitis peaked at $18 \mathrm{~h}$ following infection, but returned to near-baseline levels by $60 \mathrm{~h}$. Similarly, transferrin (Figure 1B) was significantly increased $(P<0.05)$ when compared with baseline measurements at 18,24 , and 36 h following inoculation with E. coli, but returned to prechallenge levels by $60 \mathrm{~h}$. Lactoferrin concentrations in milk increased significantly $(P<0.05)$ when compared with preinfection levels at 18, 24, 36, 48, and $60 \mathrm{~h}$ but, unlike BSA and transferrin, did not return to baseline levels during the course of the study (Figure 1C).

\section{LC-MS/MS Label-Free Quantification of Abundant Milk Proteins}

Examination of the temporal expression of milk proteins before and after experimental induction of coliform mastitis revealed changes in the relative abundance, estimated by increases in peptide counts, of proteins albumin (Figure 2A) and transferrin (Figure 2B) at 18 and $12 \mathrm{~h}$, respectively, after infusion. Peptide counts for BSA exhibited changes in relative abundance greater than 3 -fold over prechallenge levels at 12, 18, 24, 36, and $48 \mathrm{~h}$ following infection. Fewer than 5 peptides were consistently identified for transferrin, and thus no relevance was attributed to calculated fold changes across time points. Peptide counts for lactoferrin (Figure 2C) revealed a gradual increase in relative abundance with time. As with transferrin, fewer than 5 peptides were assigned to lactoferrin at all time points and thus no relevance of fold changes was assigned.

Peptide counts for the whey proteins $\beta$-LG and $\alpha$-LA decreased following infection, with the largest decrease evident between 12 and $36 \mathrm{~h}$ following infection, which corresponded to increases in BSA (Figure 2D). Changes in relative abundance of $\beta-\mathrm{LG}$ and $\alpha-\mathrm{LA}$, as determined by peptide counts, were not greater than 3 -fold com- 
pared with preinfection levels. Likewise, abundance of $\alpha_{\mathrm{S} 1}-\mathrm{CN}, \beta-\mathrm{CN}$, and $\kappa-\mathrm{CN}$ remained relatively unchanged throughout the course of the mastitis infection, and no significant differences in relative abundance across time points were detected (Figure 2E).

\section{LC-MS/MS Label-Free Quantification Corresponds to ELISA Data}

The comparison of changes in milk concentrations of BSA following experimental induction of coliform mastitis as measured by ELISA and label-free LC-MS/MS quantification levels revealed significant differences from prechallenge levels at 18 and $24 \mathrm{~h}$ for ELISA data and at $12,18,24,36$, and $48 \mathrm{~h}$ for label-free quantification data based on fold change (Figure 3A). Additionally, temporal expression patterns of BSA determined using an ELISA and normalized peptide counts determined by LC-MS/MS were almost identical. Similar to the results found with BSA, the comparison of graphic representations of the quantification of transferrin (Figure 3B) and lactoferrin (Figure 3C) by ELISA with labelfree LC-MS/MS methods revealed similar trends.

\section{LC-MS/MS Label-Free Quantification of Low-Abundance Milk Proteins Corresponds to Clinical Data}

Temporal expression patterns of the antimicrobial proteins cathelicidin- 1 and PGRP before and after infection with $E$. coli were evaluated using normalized peptide counts for each protein per time point. Because expression of both antimicrobial proteins was not detected in all biological replicates, however, and because few peptides assignments were made, the identification of cathelicidin-1 and PGRP must be regarded as tentative. Nonetheless, peak expression of both cathelicidin-1 and PGRP occurred at $24 \mathrm{~h}$ following infection (Figure $4 \mathrm{~A}$ ), which corresponded to peak increases in milk SCS (Figure 4B). Milk SCS were significantly increased $(P$ $<0.05)$ at 24 and $48 \mathrm{~h}$ post infection.

\section{DISCUSSION}

Whey from bovine milk samples collected before infection with $E$. coli and whey from milk samples collected at $12,18,24,36,48$, and $60 \mathrm{~h}$ following infection with E. coli were digested in-solution with trypsin, separated using 1-dimensional reverse-phase liquid chromatography, and identified by peptide sequencing using MS/ MS followed by database searching. Similar to previous proteomic analyses of bovine milk (Smolenski et al., 2007; Boehmer et al., 2008; Fong et al., 2008), the abundant milk proteins BSA, $\beta-\mathrm{LG}, \alpha-\mathrm{LA}, \alpha_{\mathrm{S} 1^{-}} \mathrm{CN}, \alpha_{\mathrm{S} 2^{-}}$
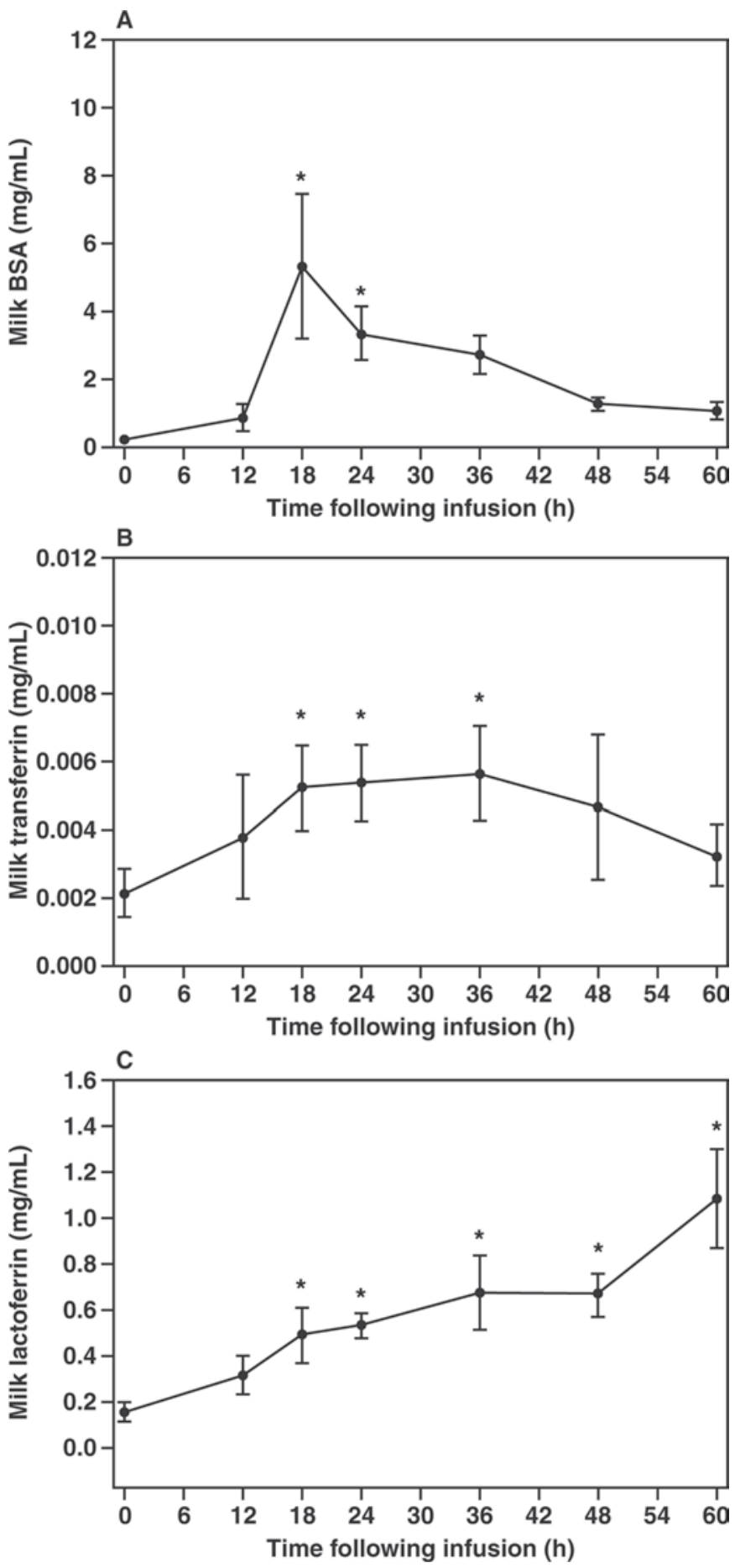

Figure 1. Results of ELISA for A) BSA, B) transferrin, and C) lactoferrin concentrations measured in milk. Mean concentrations $( \pm \mathrm{SE})$ are shown for each time point. Asterisk denotes significant $(P<0.05)$ changes relative to time zero.

$\mathrm{CN}, \beta-\mathrm{CN}$, and $\kappa$-CN were identified in whey samples collected at all time points from all 8 cows. A limited number of proteins related to host response, including 

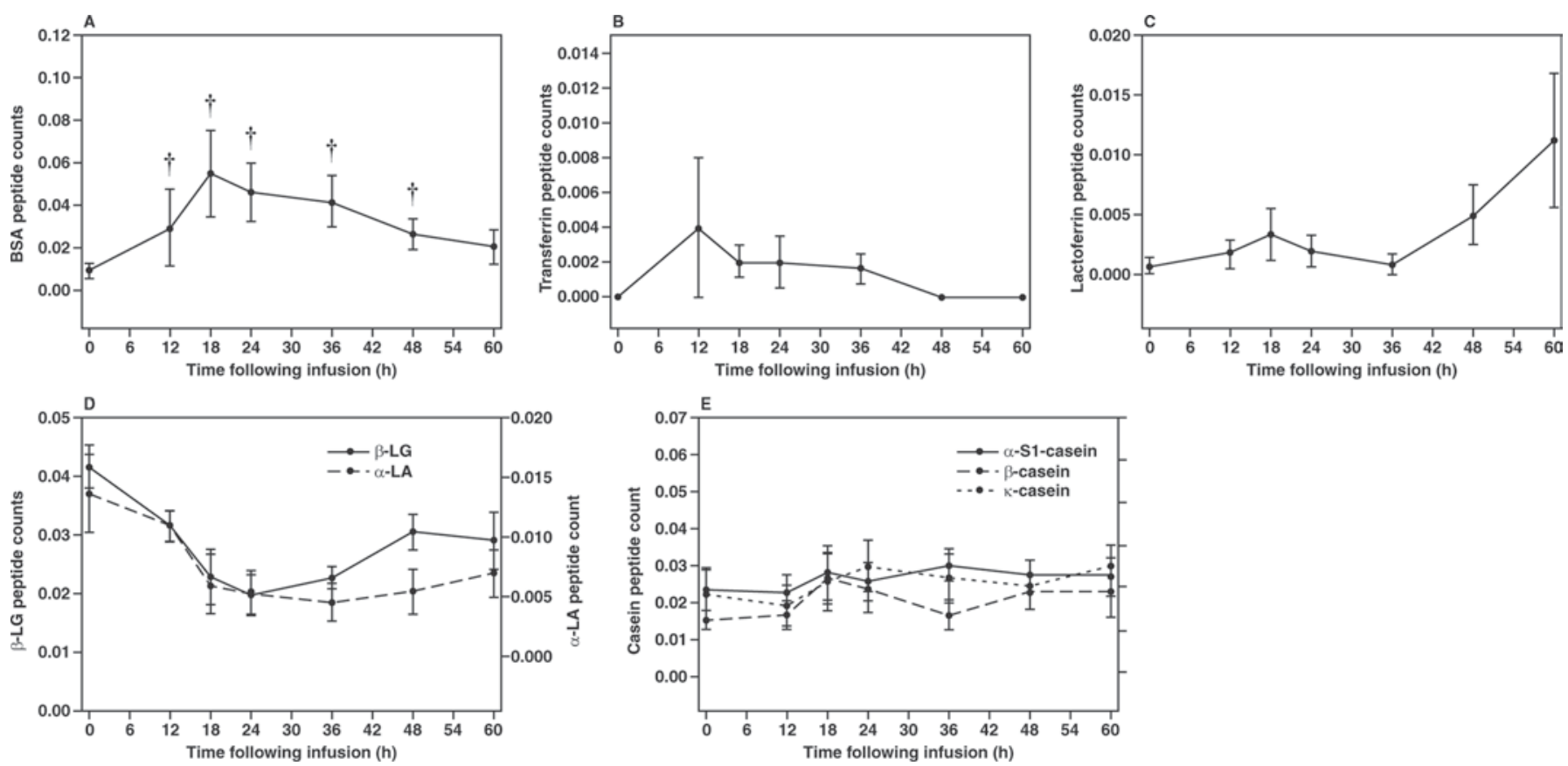

Figure 2. Normalized peptide counts for A) BSA, B) transferrin, C) lactoferrin, D) whey proteins, and E) caseins identified at each time point. Means $( \pm \mathrm{SE})$ are shown for each time point. Cross denotes 3 -fold or greater changes in peptide counts relative to time zero.

lactoferrin, transferrin, apolipoproteins AI, fibrinogen, glycosylation-dependent cell adhesion molecule-1, PGRP, and cathelicidin-1, were also identified, but discovery was in fewer than 8 cows.

In accord with earlier proteomic profiles, no sample fractionation or depletion of high-abundance proteins was performed before analysis; however, contrary to previous studies, minimal sample prep did not result in the identification of a greater number of milk proteins (Smolenski et al., 2007; Boehmer et al., 2008). The failure to detect proteins related to host response in samples from all 8 cows following infection could be attributed to the lack of sample clean-up, the use of only a 1-dimensional liquid chromatography separation of peptides before mass spectrometric analysis, or the inherent variability in the response of each cow to $E$. coli challenge. The variability in biological response to infection was evidenced by the fact that there were fluctuations in the peptide counts per protein in samples from the 8 cows used in these analyses, indicating differing levels of protein abundance, in particular for proteins related to host response (Supplemental Table 1; available online at http://www.journalofdairyscience. $\operatorname{org} /)$.

Quadrupole time-of-flight mass spectrometers are known for higher resolution capabilities compared with some other MS instruments. However, the current analyses clearly lacked the advantage of the faster scanning speed and ability to trap ions offered by the linear ion trap mass spectrometer used in previous LCMS/MS analysis of proteins related to host response (Smolenski et al., 2007), and the multidimensional fractionation of milk proteins before MS analyses performed on whey from healthy cows (Fong et al., 2008). Likewise, although dynamic exclusion (DE) was used as a means to reduce the MS/MS sampling rates of peptides from abundant milk proteins, the complexity of the matrix and the use of only a 1-dimensional liquid chromatography separation of peptides most likely resulted in the coelution of more than 5 peptides per MS scan. Only the 5 most abundant peptides in each MS scan were selected for further fragmentation via MS/ MS CID. Thus, weaker ions were most likely prevented from triggering an MS/MS event and the identification of low-abundance proteins was not possible (Spahr et al., 2000; Issaq et al., 2005; Zhang et al., 2009). The use of 2-dimensional liquid chromatography before mass spectrometric analysis, and perhaps the selection of a scan greater than 5 peptides per MS, could increase the number of low-abundance proteins identified. An additional alternative to increase the identification of low-abundance proteins would be to use a mass spectrometer with a faster scanning speed and the ability to trap or accumulate ions (Smolenski et al., 2007).

In the current analyses, the applied methods did not enable the identification of low-abundance proteins or further annotation of the bovine milk proteome. Clearly, the biological complexity of milk and the probability 
of coeluting peptides despite the use of DE affected the sensitivity of the methods. However, the secondary effect was the robust identification of peptides from a select number of highly abundant proteins that were identified in all biological replicates across all time points analyzed. Repeat injections were made on a limited number of randomly selected samples, but the number and identity of the peptides sequenced did not differ appreciably between analyses (data not shown). Because of the extended length of the gradient employed, the increased importance of biological replicates in the biomarker discovery phase, and the fact that no additional information was gained, technical replicates were not run. Despite the lack of technical replicates, the repeat identification across all time points and biological replicates of a select number of the same peptides for each abundant protein with minimal fluctuation in Mascot expect scores indicated good reproducibility of peptide counts as well as peptide sequencing.

Recent investigations have demonstrated that $\mathrm{DE}$ does not affect the accuracy of label-free quantification strategies such as peptide counts or spectral counts in assessing changes in relative protein abundance (Zhang et al., 2009), but the effects of the sample complexity as well as the lack of multi-dimensional peptide separation could skew quantification, especially for low-abundance proteins (Mueller et al., 2008; Simpson et al., 2009; Zhang et al., 2009). In the current analyses, the total peptide counts and the total spectral counts for each protein identified in each sample were almost identical, which further supports the hypothesis that the complexity of the matrix and inadequate peptide separation were the primary reasons for the apparent insensitivity of the methods because the MS/MS sampling rate of peptides from an abundant protein is expected to exceed the total number of peptides identified. Although both spectral counts and peptide counts have been shown to be correlated with relative protein abundance (Gao et al., 2003; Liu et al., 2004), spectral counts become less reliable as measures of abundance when protein identities are based on only a small number of peptide identifications (Mueller et al., 2008). Despite the patent lack of sensitivity in the current LC-MS/ MS analyses that resulted in the underestimation of spectral counts, the primary objective of assessing the accuracy of semiquantitative proteomic methods in measuring changes in the relative abundance of milk proteins during disease was accomplished using peptide counts on abundant milk proteins (Gao et al., 2003; Zhang et al., 2006).

Quantification of milk protein and inflammatory marker expression during mastitis has been limited to antibody-based strategies such as ELISA and to PCR measurements of mRNA levels (Bannerman et al.,
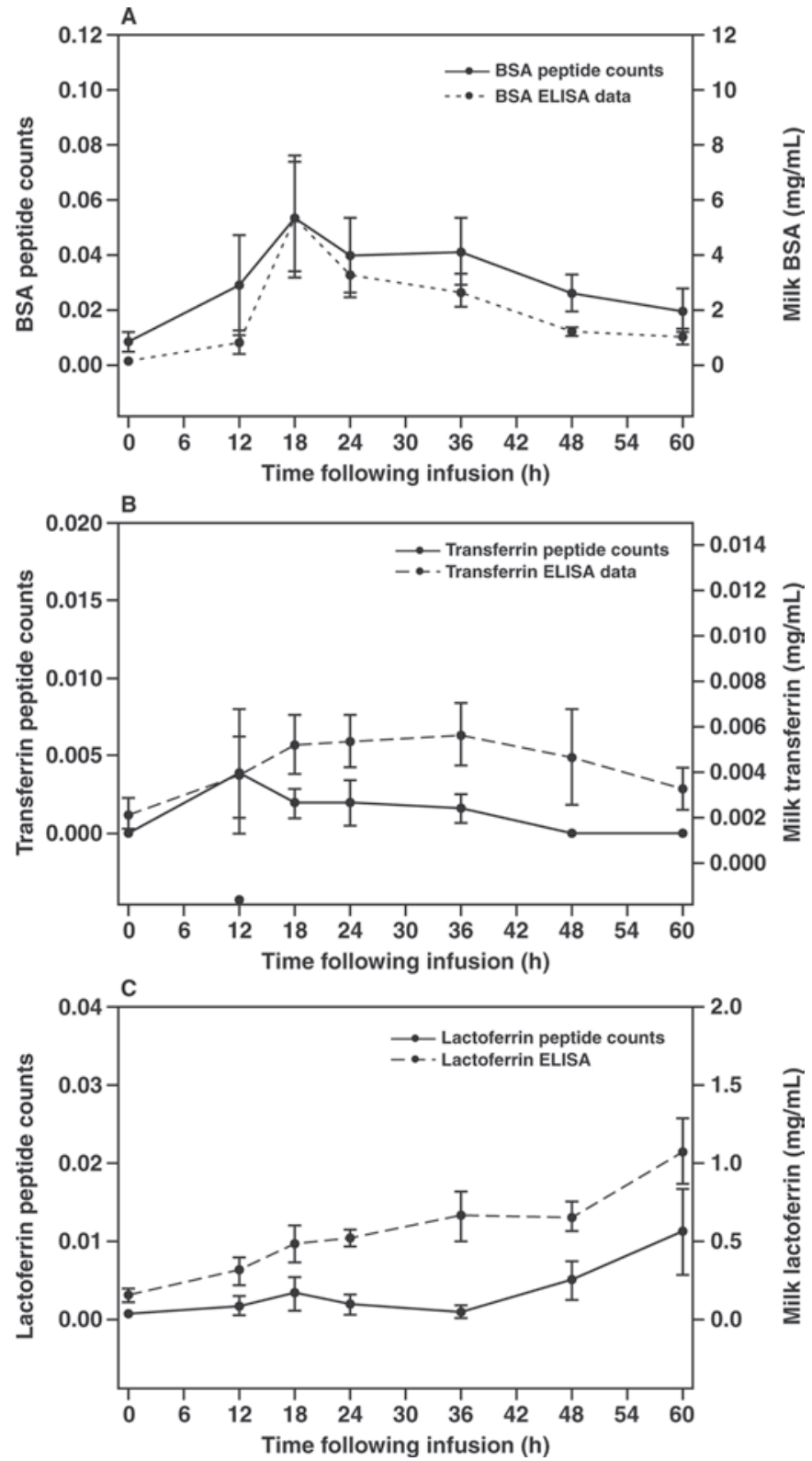

Figure 3. Comparison of normalized peptide counts and ELISA data for A) BSA, B) transferrin, and C) lactoferrin across all time points. Means $( \pm \mathrm{SE})$ are shown for each time point.

2004; McClenahan et al., 2005; Vanselow et al., 2006). As a result, a limited amount of data exists on the temporal expression of milk proteins other than soluble mediators of inflammation such as cytokines (reviewed in Bannerman, 2009) during the course of clinical mastitis. Increases in BSA have, however, been measured at several time points using an ELISA following experimental induction of coliform mastitis (Bannerman et al., 2003, 2004). The comparison of relative abundance determined using mass spectrometric label-free quantification to concentrations measured using an ELISA 

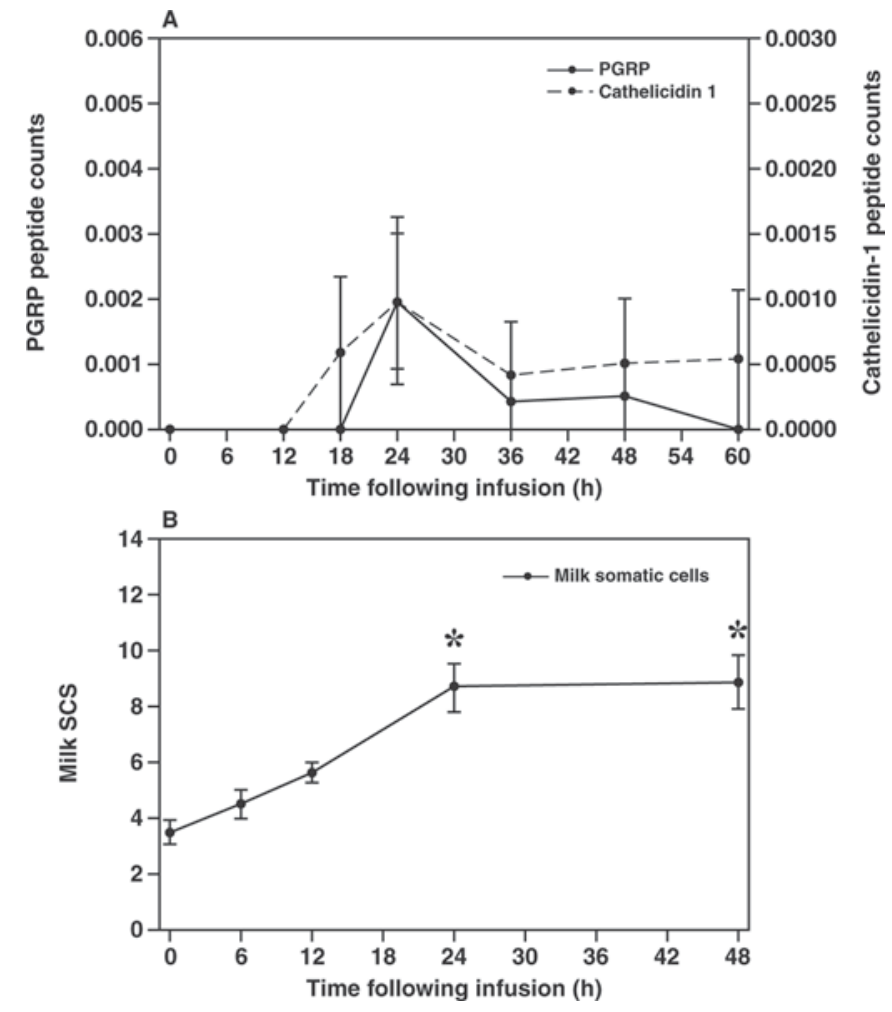

Figure 4. A) Comparison of normalized peptide counts for peptidoglycan recognition receptor protein (PGRP) and cathelicidin-1 at each time point. B) Log-transformed milk SCS at each time point. Means $( \pm$ SE $)$ are shown for each time point. Asterisk denotes significant $(P<0.05)$ changes in milk SCS relative to time zero.

have not previously been reported for milk proteins. In the current analyses, the temporal expression pattern of BSA over the course of the mastitis infection determined using normalized peptide counts indicated peak increases in milk BSA that were in accord with previous antibody-based analyses of BSA concentrations in milk during coliform mastitis (Bannerman et al., 2003, 2004). Likewise, the expression profiles of normalized peptide counts also corresponded to ELISA quantification profiles of the proteins BSA, transferrin, and lactoferrin in the milk samples collected in the current study. Additional proteins will need to be evaluated to verify the correspondence of label-free quantification with more established means of measuring protein abundance including ELISA, but results indicate the feasibility of using label-free proteomic strategies to profile changes in the abundance of milk proteins during disease.

Normalized peptide counts were also used to map the temporal expression of 2 low-abundance proteins involved in host response identified in whey following experimental induction of coliform mastitis. Because of the apparent insensitivity of the analyses and the complexity of the matrix, however, peptides from the lower abundance proteins cathelicidin-1 and PGRP, although often close to the level of detection, were not sampled in every biological replicate. Likewise, no antibodies were available for cathelicidin-1 or PGRP; thus, verification of the accuracy of the expression profile could not be accomplished by comparison to ELISA. Additionally, the total peptide counts for the antimicrobial proteins were significantly lower than the peptide counts of abundant proteins, but the peptides identified and the Mascot expectation scores of each peptide that contributed to the identification of PGRP and cathelicidin-1 were consistent across the biological replicates, as were the time points at which the proteins were identified.

Peptidoglycan recognition receptor protein is a highly conserved pattern recognition molecule involved in innate immunity that has been shown to interact with LPS and to exhibit antibacterial activity in cattle (Dziarski, 2003; Tydell et al., 2006). The cationic antimicrobial peptide cathelicidin-1 is also involved in innate immunity and is known to inactivate and destroy invading pathogens by perturbing microbial membranes (Scocchi et al., 1997). Both PGRP and cathelicidin-1 were identified in previous proteomic analyses of mastitic bovine milk (Smolenski et al., 2007; Boehmer et al., 2008), but changes in expression level during coliform mastitis were not evaluated. Neutrophils are an established source of both PGRP and cathelicidin-1 (Tydell et al., 2006; Scocchi et al., 1997) and are known to be the primary component of milk SCC during intramammary infection (Paape et al., 1981). Increases in milk SCC corresponded to increases in the relative abundance of both PGRP and cathelicidin-1 as determined by peptide counts, indicating that the proteomic data was in accord with clinical data.

The primary limitation of established quantification methods is that most are designed to measure only 1 protein in a sample at a time. Although proteomic methodologies provide the opportunity to measure an unlimited number of proteins in any given sample, investigations into quantification methods affiliated with mass spectrometry have not been initiated in studies of the bovine milk proteome. Because of issues of sensitivity, the focus of the current study was relegated to abundant milk proteins; however, profiling the temporal expression of some low-abundance proteins associated with host response was possible using LC-MS/ MS label-free quantification. Although the current study does represent the first longitudinal LC-MS/MS analyses of bovine milk samples before and after experimental induction of coliform mastitis, further research is required to address the effect of the complexity of milk and the dynamic range of bovine milk proteins, and the apparent fluctuations in biological complexity across time points following infection, on quantification 
of protein modulation during mastitis. Nonetheless, the analogous trends in temporal expression determined by the comparison of ELISA and mass spectrometric data indicate that LC-MS/MS analyses could prove useful in the evaluation of the modulation of bovine milk proteins as well as inflammatory mediators during the course of coliform mastitis. Of particular value would be the application of the label-free proteomic strategies to studies focused on the discovery of novel markers of disease in bovine milk using more sophisticated mass spectrometric analyses, and to the evaluation of milk proteins for which no bovine antibody currently exists.

\section{REFERENCES}

Bannerman, D. D. 2009. Pathogen-dependent induction of cytokines and other soluble inflammatory mediators during intramammary infection of dairy cows. J. Anim. Sci. 87:10-25.

Bannerman, D. D., M. J. Paape, W. R. Hare, and E. J. Sohn. 2003. Increased levels of LPS-binding protein in bovine blood and milk following bacterial lipopolysaccharide challenge. J. Dairy Sci. 86:3128-3137.

Bannerman, D. D., M. J. Paape, J.-W. Lee, X. Zhao, J. C. Hope, and P. Rainard. 2004. Escherichia coli and Staphylococcus aureus elicit differential innate immune responses following intramammary infection. Clin. Diagn. Lab. Immunol. 11:463-472.

Boehmer, J. L., D. D. Bannerman, K. J. Shefcheck, and J. L. Ward. 2008. Proteomic analysis of differentially expressed proteins in bovine milk during experimentally induced Escherichia coli mastitis. J. Dairy Sci. 91:4206-4218.

Bondarenko, P. V., D. Chelius, and T. A. Shaler. 2002. Identification and relative quantitation of protein mixtures by enzymatic digestion followed by capillary reversed-phase liquid chromatography - tandem mass spectrometry. Anal. Chem. 74:4741-4749.

Dziarski, R. 2003. Peptidoglycan recognition proteins (PGRPs). Mol. Immunol. 40:877-886.

Fong, B. Y., S. C. Norris, and K. P. Palmano. 2008. Fractionation of bovine whey proteins and characterization by proteomic techniques. Int. Dairy J. 18:23-46.

Gagnaire, V., J. Jardin, G. Jan, and S. Lortal. 2009. Invited review: Proteomics of milk and bacteria used in fermented dairy products: From qualitative to quantitative advances. J. Dairy Sci. 92:811825.

Gao, J., G. J. Opiteck, M. S. Friedrichs, A. R. Dongre, and S. A. Hefta. 2003. Changes in the protein expression of yeast as a function of carbon source. J. Proteome Res. 2:643-649.

Garbis, S., G. Lubec, and M. Fountoulakis. 2005. Limitations of current proteomics technologies. J. Chromatogr. A. 1007:1-18.

Hogarth, C. J., J. L. Fitzpatrick, A. M. Nolan, F. J. Young, A. Pitt, and P. D. Eckersall. 2004. Differential protein composition of bovine whey: A comparison of whey from healthy animals and from those with clinical mastitis. Proteomics 4:2094-2100.

Issaq, H. I., K. C. Chan, G. M. Janini, T. P. Conrads, and T. D. Veenstra. 2005. Multidimensional separation of peptides for effective proteomic analysis. J Chromatogr. B. 817:35-47.

Lippolis, J. D., and T. A. Reinhardt. 2008. Proteomics in animal science. J. Anim. Sci. 86:2430-2441.

Liu, H., R. G. Sadygov, and J. R. Yates III. 2004. A model for random sampling and estimation of relative protein abundance in shotgun proteomics. Anal. Chem. 76:4193-4201.

McClenahan, D. J., J. P. Sotos, and C. J. Czuprynski. 2005. Cytokine response of bovine mammary gland epithelial cells to Escherichia coli, coliform culture filtrate, or lipopolysaccharide. Am. J. Vet. Res. 66:1590-1596.

McFarland, M. A., C. E. Ellis, S. P. Markey, and R. L. Nussbaum. 2008. Proteomics analysis identifies phosphorylation-dependent
Q-synuclein protein interactions. Mol. Cell Proteomics 7:21232137.

Mosley, A. L., L. Florens, Z. Wen, and M. P. Washburn. 2009. A label free quantitative proteomic analysis of the Saccharomyces cerevisiae nucleus. J. Proteomics 72:110-120.

Mueller, L. N., M.-Y. Brusniak, D. R. Mani, and R. Aebersold. 2008. An assessment of software solutions for the analysis of mass spectrometry based quantitative proteomics data. J. Proteome Res. 7:51-61.

O'Donnell, R., J. W. Holland, H. C. Deeth, and P. Alewood. 2004. Review: Milk proteomics. Int. Dairy J. 14:1013-1023.

Old, W. M., K. Meyer-Arendt, L. Aveline-Wolf, K. G. Pierce, A. Mendoza, J. R. Sevinsky, K. A. Resing, and N. G. Ahn. 2005. Comparison of label-free methods for quantifying human proteins by shotgun proteomics. Mol. Cell Proteomics 4:1487-1502.

Paape, M. J., W. P. Wergin, A. J. Guidry, and W. D. Schultze. 1981. Phagocytic defense of the ruminant mammary gland. Adv. Exp. Med. Biol. 137:555-578

Pavelka, N., M. L. Fournier, S. K. Swanson, M. Pelizzola, P. RicciardiCastagnoli, L. Florens, and M. P. Washburn. 2008. Statistical similarities between transcriptomics and quantitative shotgun proteomics data. Mol. Cell Proteomics 7:631-644.

Reinhardt, T. A., and J. D. Lippolis. 2006. Bovine milk fat globule membrane proteome. J. Dairy Res. 73:406-416.

Reinhardt, T. A., and J. D. Lippolis. 2008. Developmental changes in the milk fat globule membrane proteome during the transition from colostrums to milk. J. Dairy Sci. 91:2307-2318.

Scocchi, M., S. Wang, and M. Zanettia. 1997. Structural organization of the bovine cathelicidin gene family and identification of a novel member. FEBS Lett. 417:311-315.

Simpson, K. L., A. D. Whetton, and C. Dive. 2009. Quantitative mass spectrometry-based techniques for clinical use: Biomarker identification and quantification. J. Chromatogr. B Analyt. Technol. Biomed. Life Sci. 877:1240-1249.

Slotta, D. J., M. A. McFarland, S. J. Makusky, and S. P. Markey. 2007. MassSieve: A new visualization tool for mass spectrometry-based proteomics. Page 75 in Proc 55th Conference on Mass Spectrometry and Allied Topics, Indianapolis, IN. American Society for Mass Spectrometry, Santa Fe, NM.

Smolenski, G., S. Haines, Y.-S. Kwan, F. Bond, J. Farr, V. Davis Sr. K. Stelwagen, and T. T. Wheeler. 2007. Characterization of host defense proteins in milk using a proteomic approach. J. Proteome Res. 6:207-215.

Spahr, C. S., S. A. Susin, E. J. Bures, J. H. Robinson, M. T. Davis, M. D. McGinley, G. Kroemer, and S. D. Patterson. 2000. Simplification of complex peptide mixtures for proteomic analysis: Reversible biotinylation of cysteinyl peptides. Electrophoresis 21:1635-1650.

Tydell, C. C., J. Yuan, P. Tran, and M. E. Selsted. 2006. Bovine peptidoglycan recognition protein-S: Antimicrobial activityl localization, secretion, and binding properties. J. Immunol. 176:1154-1162.

Vanselow, J., W. Yang, J. Herrmann, H. Zerbe, H. J. Schuberth, W. Petzl, W. Tomek, and H. M. Seyfert. 2006. DNA-remethylation around a STAT5-binding enhancer in the alpha ${ }_{\mathrm{S1}}$-casein promoter is associated with abrupt shutdown of alphas - -casein synthesis during acute mastitis. J. Mol. Endocrinol. 37:463-477.

Zhang, B., N. C. VerBerkmoes, M. A. Langston, E. Uberbacher, R. L. Hettich, and N. F. Samatova. 2006. Detecting differential and correlated protein expression in label-free shotgun proteomics. J. Proteome Res. 5:2909-2918.

Zhang, Y., Z. Wen, M. P. Washburn, and L. Florens. 2009. Effect of dynamic exclusion duration on spectral count based quantitative proteomics. Anal. Chem. 81:6317-6326.

Zybailov, B., M. K. Coleman, L. Florens, and M. P. Washburn. 2005. Correlation of relative abundance ratios derived from peptide ion chromatograms and spectrum counting for quantitative proteomic analysis using stable isotope labeling. Anal. Chem. 77:62186224 . 